\section{BMJ Paediatrics Open}

\title{
Neonatal outcomes of very low birthweight infants born to mothers with hyperglycaemia in pregnancy: a retrospective cohort study in Japan
}

Daisuke Hitaka, ${ }^{\oplus 1}$ Naho Morisaki, ${ }^{\circ 2}$ Yayoi Miyazono, ${ }^{1,3}$ Aurelie Piedvache, ${ }^{2}$ Motomichi Nagafuji, ${ }^{1}$ Syusuke Takeuchi, ${ }^{1}$ Daigo Kajikawa, ${ }^{1}$ Yu Kanai, ${ }^{1}$ Makoto Saito, ${ }^{1,3}$ Hidetoshi Takada, ${ }^{1,3}$ On behalf of the Neonatal Research Network of Japan

\section{ABSTRACT}

Objective To examine the mortality and morbidities of very low birthweight (VLBW, $<1500 \mathrm{~g})$ infants of mothers with hyperglycaemia in pregnancy.

Design and setting We conducted a retrospective cohort study using data from the Neonatal Research Network of Japan, a nationwide registry of VLBW infants (2003-2012).

Patients We studied 29626 infants born at 23 to 32 weeks without major congenital anomalies, of which 682 $(2.3 \%)$ infants were from pregnancies affected by maternal hyperglycaemia.

Main outcome measures The primary outcome was hospital mortality. Secondary outcomes were neonatal morbidities and their anthropometric values. Associations between maternal hyperglycaemia and each outcome were observed for the overall period, and statistical tests for interaction were conducted to assess whether they differed before or after the adoption of the International Association of Diabetes in Pregnancy Study Group (IADPSG) guidelines in $\mathbf{2 0 1 0}$ for the diagnosis of gestational diabetes mellitus.

Results Overall, hospital mortality $(4.1 \%$ vs $5.2 \%)$, composite outcomes of mortality and severe morbidity (54.2\% vs $60 \%$ ), and anthropometric values were not significantly different between infants of mothers with or without hyperglycaemia in pregnancy. However, the incidence of respiratory distress syndrome (RDS) in VLBW infants from mothers with hyperglycaemia was significantly higher than those from mothers without it only before (relative risk (RR) $1.09,95 \% \mathrm{Cl} 1.00$ to 1.19) and not after (RR $0.97,95 \% \mathrm{Cl} 0.83$ to 1.11 ) the adoption of the IADPSG guidelines.

Conclusion VLBW infants born to mothers with hyperglycaemia in pregnancy do not seem to be at higher risk of mortality and morbidities, except for RDS only before the adoption of the IADPSG guidelines.

\section{INTRODUCTION}

Maternalhyperglycaemiain pregnancyisamajor complication, known to lead to other serious complications such as intrauterine fetal death, fetal macrosomia, neonatal hypoglycaemia, jaundice, polycythaemia, hypocalcaemia ${ }^{1-3}$ and

\section{What is known about the subject?}

The incidence of respiratory distress syndrome (RDS) in term infants from mothers with hyperglycaemia in pregnancy is greater than infants from those not affected by it.

- Afew studies have reported the mortality and morbidities of very low birthweight (VLBW) infants from mothers with hyperglycaemia in pregnancy.

- No study has observed how change in the diagnostic criteria of gestational diabetes mellitus influences the association between maternal hyperglycaemia and mortality and morbidities of VLBW infants.

\section{What this study adds?}

As a whole, no significant difference was observed in mortality, severe morbidity or anthropometric values between VLBW infants with and without maternal hyperglycaemia in pregnancy.

- VLBW infants born to mothers with hyperglycaemia in pregnancy showed a greater risk of RDS only before the adoption of the International Association of Diabetes in Pregnancy Study Group guidelines.

preterm birth. ${ }^{4}$ Depending on the population studied and the diagnostic tests employed, this complication is reported to occur in $2 \%$ to $25 \%$ of all pregnancies. ${ }^{5}$

The risk of preterm birth in mothers with diabetes is several times higher than that in mothers without diabetes, ${ }^{6}$ and very preterm births themselves have a high risk of death and severe morbidity in the first place. ${ }^{7}$

Furthermore, the effect of diabetes on the child's health may differ by the severity of the diabetes and the impact of glycaemic control mode on infant outcomes. ${ }^{8}$ There have been only a few studies to examine the association between hyperglycaemia in pregnancy 
and the mortality and morbidities of the premature infants. ${ }^{8-12}$ Several studies had reported that very low birthweight (VLBW, $<1500 \mathrm{~g}$ ) infants born to mothers with hyperglycaemia in pregnancy were at higher risk of necrotising enterocolitis (NEC), ${ }^{8} 11$ though others had shown not. ${ }^{91012}$ A recent study conducted in seven countries (including Japan) suggested that hyperglycaemia in pregnancy is not associated with an increased risk of in-hospital mortality or severe morbidity in VLBW infants. ${ }^{12}$ However, the population combined those diagnosed with gestational diabetes mellitus (GDM) from both before (2007-2010) and after (2010-2015) the publication of the International Association of Diabetes in Pregnancy Study Group (IADPSG) criteria for GDM in 2010. The influence of the change in criteria for diagnosis is non-negligible; for instance, in Japan, after the adoption of the IADPSG criteria for GDM in 2010, ${ }^{13}$ which resulted in relaxation of the criteria for diagnosis, frequency of GDM pregnancies increased twofold to fourfold to $6 \%-12 \% .^{1415}$

Thus, the purpose of this study was to exsamine the association between maternal hyperglycaemia in pregnancy and mortality, morbidities, birth weight for gestational age and extrauterine growth of VLBW infants and investigate whether this association differed before and after the adoption of the IADPSG criteria for GDM diagnosis. Japan has one of the lowest neonatal mortality and morbidity rates among the developed countries, ${ }^{16}$ and the findings of this study would be useful internationally to understand the impact of maternal hyperglycaemia in pregnancy on preterm infants in an advanced perinatal care.

\section{METHODS}

\section{Study population}

Patient data from the Neonatal Research Network of Japan (NRNJ), which contain prospectively registered data on VLBW infants admitted to the 204 participating neonatal intensive care units (NICUs) nationwide were analysed. ${ }^{17}$ All institutes registered individual data, including clinical characteristics and morbidities, for the VLBW infants under their care. Data from the period of 1 January 2003 to 31 December 2012 were examined. The data consisted of VLBW infants from $96 \%$ (72/75 as of 2008) of all level 3 NICUs and from seven level 2 NICUs in Japan. ${ }^{18}$

Infants born before 23 weeks and after 33 weeks were excluded from the analysis, as the former have a high mortality rate and almost all of the latter were small for gestational age (SGA), which has a strong impact on mortality and morbidity. We also excluded VLBW infants born between 23 and 32 weeks with congenital anomalies (serious congenital heart disease or major genetic disorder), those who died at the delivery room, and those with missing data on maternal diabetes, or missing data on their characteristics and outcomes.

\section{Definition of disease and outcomes}

For each infant, the NRNJ database collects data on maternal background (including age, parity) and infant outcomes (including birth anthropometrics, whether or not specific complications had developed during hospitalisation). A database manual was distributed to each hospital to ensure the definition of variables was uniform across the participating institutions.

Neonatal morbidities recorded in the database included: respiratory distress syndrome (RDS), patent ductus arteriosus (PDA), necrotising enterocolitis (NEC), intraventricular haemorrhage (IVH), periventricular leukomalacia (PVL), chronic lung disease (CLD) (measured at 28 days of age and at 36 weeks) and retinopathy of prematurity (ROP). From these data, we defined the composite outcome as the infant experiencing any of the following: hospital death, requiring PDA treatment (indomethacin or ligation), NEC, IVH grades III-IV, PVL, CLD at 36 weeks or requiring $\mathrm{ROP}$ treatment (laser photocoagulation or antivascular endothelial growth factor).

The diagnosis criteria of all complications were predefined in the distributed manual as follows: RDS was diagnosed based on clinical presentation and radiographic findings; PDA was diagnosed based on both echocardiographic and clinical findings; NEC was defined as Bell stage II or greater; IVH was diagnosed based on cranial ultrasound and graded using the classification of Papile $e t a l^{19}$; PVL was diagnosed based on cranial ultrasound or MRI under the presence of cystic lesion around periventricular white matter; CLD 28 and CLD 36 were defined as the need for supplemental oxygen at 28 days of age or at 36 weeks of postmenstrual age, respectively; and ROP was staged according to the classification defined by the Japanese Ministry of Health, Labour and Welfare, which directly correlates with ICROP (International Classification of Retinopathy of Prematurity).

From the birth and discharge anthropometrics included in the database, z-scores for infant weight, height and head circumference were calculated using neonatal anthropometric charts-reference data of vaginal deliveries during 2003-2005 in Japan..$^{20}$ SGA was defined as birth weight being less than the 10th percentile for the gestational age based on the same reference data. Change in z-scores for each measurement from birth to discharge was calculated by subtracting the z-scores from each other.

Data on the presence of glucose intolerance during pregnancy were collected throughout the period, as a yes/no question. In Japan, the random blood glucose test is used for screening of GDM during the first trimester. Then, between 24 and 28 weeks of gestation, either the random blood glucose or glucose challenge test $(50 \mathrm{~g})$ were performed for screening. These protocols did not change throughout the study period. However, in 2010, GDM diagnosis criteria changed in Japan, when the Japan Society of Obstetrics and Gynecology (JSOG) adopted the IADPSG criteria. ${ }^{13}$ Before this adoption, diagnosis of GDM was determined according to the JSOG criteria, in which two or more abnormal values of the following were required in the $75 \mathrm{~g}$ oral glucose tolerance test (OGTT): fasting blood glucose $\geq 100 \mathrm{mg}$ / $\mathrm{dL}(\geq 5.6 \mathrm{mmol} / \mathrm{L}) ; 1$ hour $\geq 180 \mathrm{mg} / \mathrm{dL}(\geq 10.0 \mathrm{mmol} / \mathrm{L})$ 
or 2 hours $\geq 150 \mathrm{mg} / \mathrm{dL}(\geq 8.3 \mathrm{mmol} / \mathrm{L})$. After adopting the IADPSG criteria, diagnosis of GDM was based on one of the following glucose levels in the $75 \mathrm{~g}$ OGTT: fasting blood glucose $\geq 92 \mathrm{mg} / \mathrm{dL}(\geq 5.1 \mathrm{mmol} / \mathrm{L}) ; 1$ hour $\geq 180 \mathrm{mg} / \mathrm{dL}(\geq 10.0 \mathrm{mmol} / \mathrm{L})$ or 2 hours $\geq 153 \mathrm{mg} / \mathrm{dL}$ $(\geq 8.5 \mathrm{mmol} / \mathrm{L})$. A previous study showed that this change increased the prevalence of GDM. ${ }^{14}$ Thus, 2003-2009 was defined as the pre-IADPSG phase and 2011-2012 as the post-IADPSG phase, and we categorised the infants based on birth year accordingly. Data on the presence of maternal glucose intolerance during pregnancy were recorded except for all of the following items: subtype of diabetes mellitus (DM) (GDM, overt DM in pregnancy or pre-existing DM), status of glycaemic control and presence of treatment and its content.

\section{Patient involvement}

Patients were not directly involved in the design of this study.

\section{Statistical analysis}

First, the background characteristics of the mothers and infants were described using proportions for categorical variables and means with SD for continuous variables. Categorical variables were compared with $\chi^{2}$ test and continuous variables with two-sample t-test.

Second, to evaluate the association between hyperglycaemia in pregnancy and dichotomous neonatal outcomes, Poisson regression analyses were performed to calculate adjusted relative risk (RR) and 95\% CIs. Multivariate linear regression analyses were performed to calculate coefficients and 95\% CIs for continuous outcomes. For these analyses, based on clinical relevance, the following variables were introduced into the models as confounders: maternal age, parity, mode of delivery, use of antenatal steroid, sex of the infants, gestational week, SGA and Apgar score at $5 \mathrm{~min}$. Maternal ages were left continuous and investigated non-linearities using cubic spline regression model. As a large number of anthropometric values at discharge were missing $(n=9754,11446$, 11454 for weight, height and head circumference, respectively), multiple imputation methods were carried out with chained equations to impute the missing values with exclusion of missing data from infants discharged after 41 weeks of gestational age which were unsuitable for predicting anthropometric z-scores accurately. Complete case analysis, as well as analyses not including variables which could act as mediators (SGA, Apgar score at $5 \mathrm{~min}$ ) was also done as sensitivity analysis.

Third, to assess whether the association differed between the pre-IADPSG and post-IADPSG phases, statistical tests for interaction between the main effect and time period were conducted using multivariate logistic regression analysis of each outcome, and stratified analyses were conducted if significant or close to significant.

In all analyses, a two-sided p-value $<0.05$ was considered statistically significant with the exception of p-value

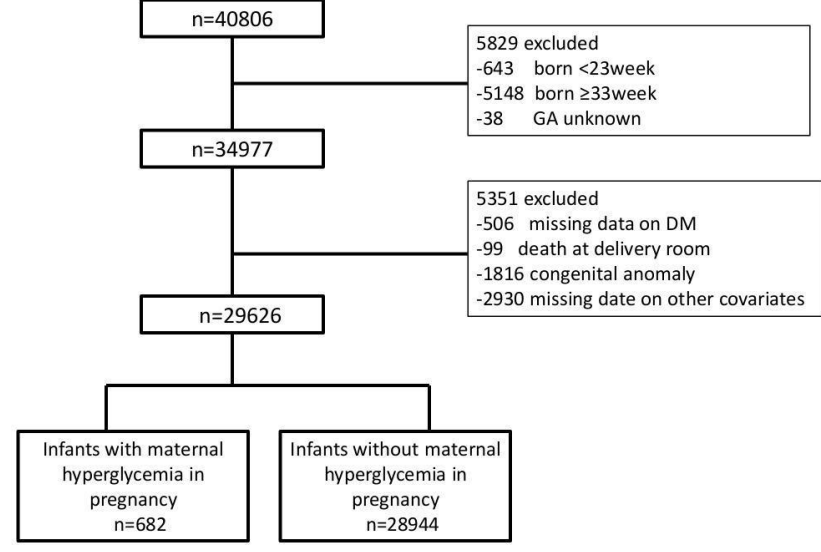

Figure 1 Population flow chart. DM, diabetes mellitus; GA, gestational age.

for interaction $<0.1$. Stata V.14.0 (College Station, Texas, USA) was used for all analyses.

\section{Ethics statement}

This study was approved by the Internal Review Board of Tokyo Women's Medical University, where all data were collected and stored. Written informed consent was obtained from the parents or guardians.

\section{RESULTS}

Figure 1 shows the population of this study. The NRNJ cohort was composed of 40806 VLBW infants born during the study period. Of these, 34977 infants were born at 23 to 32 weeks of gestation. Of these, 506 infants were excluded because of missing data on maternal diabetes, 99 infants were excluded because of death at delivery room, 1816 infants were excluded because of congenital anomaly and 2930 infants were excluded due to missing data on their characteristics and outcomes. Finally, 29626 infants were included in the analysis.

The characteristics of the mothers and infants included in this study are shown in table 1 . Mothers with hyperglycaemia in pregnancy was significantly older than mothers without it $(p<0.001)$. Gestational week and median birth weight in infants of mothers with hyperglycaemia in pregnancy were significantly higher than that of mothers without it ( $\mathrm{p}=0.03, \mathrm{p}<0.01$, respectively). Values of weight and length at discharge in infants of mothers with hyperglycaemia in pregnancy were higher than those of mothers without it $(\mathrm{p}<0.01, \mathrm{p}<0.05$, respectively) except for head circumference $(p=0.53)$. The proportions of singleton, caesarean delivery, use of antenatal steroids, male (sex), and SGA, and Apgar scores were not significantly different between the two groups.

Table 2 compares the mortality and morbidities between the two groups. There was no significant difference between the two groups in regard to any morbidity either in the crude analysis or after adjusting for confounders as previously described.

Statistical association between hyperglycaemia in pregnancy and incidence of RDS and ROP of the infants was 
Table 1 Maternal and infant characteristics of 29626 very low birthweight infants

\begin{tabular}{|c|c|c|c|}
\hline Characteristics & $\begin{array}{l}\text { Infants with maternal } \\
\text { hyperglycaemia in pregnancy } \\
\text { ( } N=682)\end{array}$ & $\begin{array}{l}\text { Infants without maternal } \\
\text { hyperglycaemia in pregnancy } \\
\text { ( } N=28944)\end{array}$ & $P$ value \\
\hline \multicolumn{4}{|l|}{ Maternal } \\
\hline Maternal age (SD) & $33.8 \pm 4.8$ & $31.2 \pm 5.8$ & $<0.01$ \\
\hline Maternal age, missing, $\mathrm{n}(\%)$ & $8(1.2)$ & $803(2.8)$ & \\
\hline Singleton, n (\%) & $501(73.5)$ & $21652(74.5)$ & 0.42 \\
\hline Caesarean delivery, $\mathrm{n}(\%)$ & $552(80.9)$ & $22694(78.4)$ & 0.11 \\
\hline Antenatal steroids, n (\%) & $318(46.6)$ & $14223(49.1)$ & 0.20 \\
\hline \multicolumn{4}{|l|}{ Neonatal } \\
\hline Male, n (\%) & $375(55.0)$ & $14896(51.4)$ & 0.07 \\
\hline SGA, n (\%) & $222(32.6)$ & $9458(32.7)$ & 0.33 \\
\hline Apgar score at $1 \mathrm{~min}$, mean (SD) & $5.3 \pm 2.4$ & $5.3 \pm 2.4$ & 0.49 \\
\hline Apgar score at 5 min, mean (SD) & $7.4 \pm 1.9$ & $7.3 \pm 1.9$ & 0.20 \\
\hline Apgar score at $5 \mathrm{~min}<7, \mathrm{n}(\%)$ & $164(24.1)$ & 7588 (26.2) & 0.20 \\
\hline Gestational age (weeks), mean (SD) & $28.1 \pm 2.5$ & $27.9 \pm 2.5$ & 0.03 \\
\hline Birth weight (g) & $1045 \pm 286$ & $1010 \pm 293$ & $<0.01$ \\
\hline \multicolumn{4}{|l|}{ Measurement at discharge } \\
\hline Weight (g) & $2798 \pm 880(n=670)$ & $2716 \pm 850(n=27406)$ & $<0.01$ \\
\hline Length (cm) & $46.6 \pm 4.9(n=633)$ & $46.2 \pm 4.9(n=25503)$ & $<0.05$ \\
\hline Head circumference $(\mathrm{cm})$ & $34.3 \pm 3.1(n=641)$ & $34.2 \pm 3.1(n=25616)$ & 0.53 \\
\hline
\end{tabular}

SGA, small for gestational age.

almost close to significant between infants born in the pre-IADPSG and post-IADPSG phase (p-value for interaction $=0.10,0.12$, respectively) (table 3 ). For both outcomes, stratified analyses by study period were performed. As a result, for those born in the pre-IADPSG phase, infants of mothers with hyperglycaemia in pregnancy had higher rates of RDS (RR 1.09, 95\% CI 1.00 to 1.19) than infants of mothers without it. This difference was largely affected by infants born at 28 to 29 weeks of gestation (online supplementary figure). For those born in the post-IADPSG phase, there was no significant difference in the incidence of
RDS between infants of mothers with and without hyperglycaemia in pregnancy (RR $0.97,95 \%$ CI 0.83 to 1.11 ). Regarding the incidence of ROP, there was no significant difference for those born in the pre-IADPSG and postIADPSG phase (RR $1.11,95 \%$ CI 0.87 to 1.42 and RR 0.85 , $95 \%$ CI 0.64 to 1.11 , respectively).

Table 4 shows the distribution of anthropometric z-scores for infants from mothers with and without hyperglycaemia in pregnancy, after multiple imputations. Z-scores of weight, height and head circumference, as well as change in z-scores for all three measures were

Table 2 Mortality and morbidities among infants with or without maternal hyperglycaemia in pregnancy

\begin{tabular}{|c|c|c|c|c|}
\hline Outcomes, n (\%) & $\begin{array}{l}\text { Infants with maternal } \\
\text { hyperglycaemia in } \\
\text { pregnancy }(n=682)\end{array}$ & $\begin{array}{l}\text { Infants without maternal } \\
\text { hyperglycaemia in } \\
\text { pregnancy }(n=28944)\end{array}$ & $P$ value & $\begin{array}{l}\text { Adjusted* RR } \\
\text { (95\% CI) }\end{array}$ \\
\hline Hospital death & $28(4.1)$ & $1504(5.2)$ & 0.20 & 0.83 (0.58 to 1.20$)$ \\
\hline RDS & $438(64.2)$ & $17842(61.6)$ & 0.17 & 1.04 (0.99 to 1.1$)$ \\
\hline NEC & $9(1.5)$ & $440(1.5)$ & 0.67 & $0.92(0.48$ to 1.77$)$ \\
\hline PDA required any treatment & $250(36.7)$ & $10964(37.9)$ & 0.52 & $1.00(0.92$ to 1.1$)$ \\
\hline IVH grades III-IV or PVL & $47(6.9)$ & $2328(8.0)$ & 0.27 & $0.86(0.65$ to 1.15$)$ \\
\hline CLD 28 & $236(34.6)$ & $10822(37.4)$ & 0.13 & $0.97(0.89$ to 1.06$)$ \\
\hline ROP required any treatment & $101(14.8)$ & $4662(16.1)$ & 0.36 & 0.99 (0.84 to 1.18$)$ \\
\hline Composite† & 370 (54.2) & $16205(60.0)$ & 0.37 & 1.00 (0.95 to 1.07$)$ \\
\hline
\end{tabular}

*Adjusted for maternal age, parity, mode of delivery, sex, antenatal steroid use, gestational week, SGA and Apgar score at 5 min. †Includes hospital death, NEC, PDA required any treatment, IVH or PVL, CLD 36 and ROP required any treatment.

CLD, chronic lung disease; IVH, intraventricular hemorrhage; NEC, necrotizing enterocolitis; PDA, patent ductus arteriosus; PVL, periventricular leukomalacia; RDS, respiratory distress syndrome; ROP, retinopathy of prematurity. 
Table 3 Interaction between maternal hyperglycaemia in pregnancy and periods (pre-IADPSG and post-IADPSG) on several neonatal outcomes

\begin{tabular}{ll}
\hline Outcomes & $\begin{array}{l}\text { P-value for } \\
\text { interaction* }\end{array}$ \\
\hline Hospital death & 0.61 \\
RDS & 0.1 \\
NEC & 0.88 \\
PDA required any treatment & 0.68 \\
IVH or PVL & 0.19 \\
CLD 28 & 0.61 \\
ROP required any treatment & 0.12 \\
Composite & 0.80 \\
\hline
\end{tabular}

${ }^{*}$ Calculated using multivariate logistic regression analysis with adjustment for maternal age, parity, mode of delivery, sex, antenatal steroid use, gestational week, SGA, Apgar score at 5 min.

$\dagger P$ value for interaction $<0.1$ is considered significant. łIncludes hospital death, NEC, PDA required any treatment, IVH or $P V L, C L D 36$ and ROP requiring any treatment.

CLD, chronic lung disease; IVH, intraventricular hemorrhage; NEC, necrotizing enterocolitis; PDA, patent ductus arteriosus; PVL, periventricular leukomalacia; RDS, respiratory distress syndrome; ROP, retinopathy of prematurity.

not significantly different between the two groups. The sensitivity analyses, when using complete cases (and not conducting multiple imputations) of the results were not significantly different in all measurements.

\section{DISCUSSION}

In this study, we examined the association between maternal hyperglycaemia in pregnancy and mortality and morbidities of VLBW infants based on a large, nationwide Japanese database. The present study demonstrated that maternal hyperglycaemia in pregnancy has no impact on the outcomes for VLBW infants except for RDS. Furthermore, the increase in risk of RDS was only significant for pregnancies with maternal hyperglycaemia in pregnancy diagnosed according to the previous guidelines.

To the best of our knowledge, to date, five studies have examined the association between maternal hyperglycaemia in pregnancy and mortality and morbidities of VLBW infants. Of the five studies, three reported that maternal hyperglycaemia in pregnancy was unrelated to any major complications for VLBW infants. ${ }^{9} 1012$ On the other hand, the other two studies showed that VLBW infants born to mothers with hyperglycaemia in pregnancy had a higher incidence of NEC. ${ }^{811}$ In the most recent and largest study to date, Persson et $a l^{12}$ reported that the risk of a variety of adverse outcomes (NEC, bronchopulmonary dysplasia, ROP treatment, treated PDA, IVH grades III-IV, cystic PVL, RDS and in-hospital mortality) or their composite outcome did not differ between infants of mothers with or without diabetes. With the exception of RDS, these insignificant findings might be acceptable since associations between other severe morbidities and maternal hyperglycaemia were not universally noted.

It is generally believed that inadequate control of hyperglycaemia in pregnancy exposes the infant to the risk of RDS, as insulin inhibits gene expressions of surfactant proteins A and B in lung epithelial cells. ${ }^{22-24}$ Mature infants born to mothers with diabetes has shown to carry a sixfold increase in risk of RDS, consistent with this hypothesis. ${ }^{25}$ However, all five previous studies on VLBW failed to find a significant association between maternal hyperglycaemia in pregnancy and the risk of RDS. Our study is the first to demonstrate that the incidence of RDS increased with maternal hyperglycaemia in pregnancy, but this association was present only in those born before the diagnostic criteria of GDM was changed in 2010.

Our finding that increased risk of RDS was observed only in infants of mothers with hyperglycaemia in pregnancy diagnosed before the relaxation of the GDM diagnostic criteria in 2010 suggests that only the more severe cases of maternal hyperglycaemia in pregnancy carry higher risk of RDS in infants. This finding is consistent with previous reports on mature infants, where RDS risk was found to be highest among those with the most severe cases of diabetes, namely, unstable type 1 diabetes. ${ }^{25}$ Our study may have had the power to detect the association between hyperglycaemia in pregnancy and RDS due to its relatively large sample size and our homogeneous definition of GDM used. In comparison, a recent large-scale

Table 4 Growth measure z-scores among infants with or without maternal hyperglycaemia in pregnancy (multiple imputation used to impute missing values $n=29626$ )

\begin{tabular}{llll}
\hline & $\begin{array}{l}\text { Infants with maternal } \\
\text { hyperglycaemia in pregnancy }\end{array}$ & $\begin{array}{l}\text { Infants without maternal } \\
\text { hyperglycaemia in pregnancy }\end{array}$ & P value* $^{*}$ \\
\hline Weight z-score at discharge & -1.26 & -1.39 & 0.19 \\
Change in weight z-score & -0.58 & -0.65 & 0.2 \\
Length z-score at discharge & -1.54 & -1.61 & 0.35 \\
Change in length z-score & -1.17 & -1.17 & 0.35 \\
Head circumference z-score at discharge & 0.39 & 0.22 & 0.2 \\
Change in head circumference z-score & 0.34 & 0.26 & 0.49 \\
\hline
\end{tabular}

*Multivariate logistic regression analysis with adjustment for maternal age, parity, mode of delivery, sex, antenatal steroid use, gestational week and SGA.

SGA, small for gestational age. 
analysis, conducted across seven countries, which failed to detect this association, had inconsistencies in its diagnostic criteria to assess GDM.

Interestingly, although about half of the infants in this study did not receive antenatal steroids which may help mature the lung, the rate of RDS among infants of mothers with diabetes $(64 \%)$ was comparable to other populations where only about $20 \%$ did not receive antenatal steroids. ${ }^{9-11}$ While this is only a speculation, this discrepancy in the incidence of RDS may be attributable to the difference in risk of RDS by race: in more mature infants (34 to 42 weeks of gestational age), Asian infants have been reported to have a lower risk for RDS than Caucasian infants. ${ }^{26}$

It is universally known that infants born at term from mothers with diabetes tend to have larger birth weights (unless the disease is severe); however, anthropometric data of preterm infants of maternal diabetes is lacking. In our study, birth weight in VLBW infants of maternal hyperglycaemia in pregnancy was significantly higher than those in the control group. Our study findings differed from those from Boghossian who reported that extremely preterm infants of mothers using insulin before pregnancy were smaller at birth (height and head circumference, but not weight) than mothers who do not use insulin before pregnancy, which the author attributed to prolonged hyperglycaemia causing deterioration of vascular condition. ${ }^{8}$ Most of the macrosomia of infants from mothers with diabetes develops in the third trimester, when maternal lipids as well as glucose produce increased fetal adiposity. Comparison of Boghossian's study and ours is difficult due to the paucity of detailed information on the types of DM and treatment in our study. One possible explanation to the difference in findings may be differences in prevalence of insulin therapy. Although we do not have the exact proportion, considering the general prevalence of pregestational DM in Japan $(<3 \%)$, it is likely that most women included in our study were not receiving insulin treatment prior to pregnancy. Our findings generate a hypothesis that mothers with hyperglycaemia in pregnancy who do not require insulin before pregnancy deliver larger weight infants even if the delivery is very preterm, while those with more severe diabetes, who require the use of insulin before pregnancy, the infants tend to be smaller. Further research should be conducted on this topic.

We acknowledge that there are several limitations to this study. First, we did not have data on the type and onset of DM, status of glycaemic control, presence and details of treatment. Furthermore, the mothers were assessed their glycaemic status at one time point between 24 and 28 weeks of gestation, and thus we were not able to account for the increase in risks of hyperglycaemia in proportion to its duration. These paucity of information on maternal diabetes might lead to insufficient assessment of some risks such as NEC, ${ }^{8}$ in which Boghossian reported that preterm infants born to mothers with insulin use before pregnancy had higher risk of NEC than infants born to mothers with insulin use started during pregnancy and without insulin use. Second, our study population was limited to preterm infants admitted to the NICU, thus excluding delivery room deaths, infants with congenital anomalies and infants born at term. Thus, we were not able to evaluate disorders of infants of mothers with diabetes, such as hyperbilirubinaemia, polycythaemia, hypocalcaemia and asymmetric cardiac septal hypertrophy. Third, our study had a high proportion of missing data on anthropometric values at discharge. Although we conducted imputation to account for the missing data, we cannot rule out the chance of bias.

Despite these limitations, our findings can be interpreted as revealing the overall trend in regard to results of VLBW infants born to mothers with hyperglycaemia in pregnancy, as in Asian women, GDM accounts for the majority of occurrences of hyperglycaemia in pregnancy. ${ }^{27}$

In conclusion, from our study, which included a large number of VLBW infants of mothers with hyperglycaemia in pregnancy, we found that there is no relation between hyperglycaemia in pregnancy and in-hospital mortality and risk of severe morbidities. Nevertheless an association between risk of RDS and hyperglycaemia in pregnancy differed according to the criteria used for GDM diagnosis. We found that before the IADPSG guidelines for GDM diagnosis was adopted in 2010, VLBW infants born to mothers with hyperglycaemia in pregnancy had an increased risk of RDS.

Acknowledgements The authors thank T Mayers (Medical English Communications Center, University of Tsukuba) for grammatical review and advice.

Collaborators Institutions enrolled in the study of the Neonatal Research Network, Japan, were as follows: Sapporo City General Hospital, Asahikawa Kosei General Hospital, Engaru-Kosei General Hospital, Kushiro Red Cross Hospital, ObihiroKosei General Hospital, Tenshi Hospital, NTT Higashinihon Sapporo Hospital, Nikko Memorial Hospital, Nayoro City General Hospital, Sapporo Medical University, Asahikawa Medical University, Aomori Prefectural Central Hospital, Iwate Medical University, Iwate Prefectural Ofunato Hospital, Iwate Prefectural Kuji Hospital, Iwate Prefectural Ninohe Hospital, Sendai Red Cross Hospital, Akita Red Cross Hospital, Tsuruoka Municipal Shonai Hospital, Yamagata University, Yamagata Prefectural Central Hospital, Fukushima Medical University, Takeda General Hospital, Fukushima National Hospital, Tsukuba University, Tsuchiura Kyodo Hospital, Ibaraki Children's Hospital, Dokkyo Medical University, Jichi Medical University, Ashikaga Red Cross Hospital, Gunma Children's Medical Center, Kiryu Kosei General Hospital, Fuji Heavy Industries Health Insurance Society Ota Memorial Hospital, Gunma University, Saitama Children's Medical Center, Nishisaitama-chuo National Hospital, Saitama Medical University Saitama Medical Center, Kawaguchi Municipal Medical Center, Jichi Medical University Saitama Medical Center, Asahi General Hospital, Chiba Kaihin Municipal Hospital, Kameda Medical Center, Tokyo Women's Medical University Yachiyo Medical Center, Juntendo University Urayasu Hospital, Tokyo Metropolitan Children's Medical Center, Tokyo Women's Medical University, Aiiku Hospital, Nihon University Itabashi Hospital, National Center for Global Health and Medicine, Tokyo Medical University, Teikyo University, Showa University, Japan Red Cross Medical Center, National Center for Child Health and Development, Tokyo Metropolitan Otsuka Hospital, Toho University, Tokyo Metropolitan Bokuto Hospital, Tokyo Jikei Medical University, Tokyo Medical and Dental University, Saint Luku's International Hospital, Juntendo University, Sanikukai Hospital, Katsushika Red Cross Hospital, Yokohama Rosai Hospital, Yokohama City University Medical Center, St. Marianna University School of Medicine Hospital, Kanagawa Children's Medical Center, Tokai University, Kitazato University, Odawara Municipal Hospital, Nippon Medical School Musashi Kosugi Hospital, Saiseikai Yokohamashi Tobu Hospital, National Hospital Organization Yokohama Medical Center, Yamanashi Prefectural Central Hospital, Nagano Children's Hospital, Shinshu University, lida Municipal Hospital, National Hospital Organization Shinshu Ueda Medical Center, Saku General Hospital, Niigata University, Niigata Prefectural Central Hospital, Niigata Municipal Hospital, Nagaoka Red Cross Hospital, Koseiren Takaoka Hospital, Toyama Prefectural Central Hospital, Toyama University, Ishikawa Medical Center for Maternal and Child Health, Kanazawa Medical University, Kanazawa Medical 
Center, Fukui Prefectural Hospital, Fukui University, Gifu Prefectural General Medical Center, National Hospital Organization Nagara Medical Center, Takayama Red Cross Hospital, Seirei Hamamatsu Hospital, Shizuoka Saiseikai Hospital, Shizuoka Children's Hospital, Hamamatsu Medical University, Numazu Municipal Hospital, Yaizu City Hospital, Fujieda Municipal General Hospital, Nagoya Red Cross Daini Hospital, Nagoya University, Nagoya Red Cross Daiichi Hospital, Toyohashi Municipal Hospital, Nagoya City West Medical Center, Anjo kosei Hospital, Tosei General Hospital, Komaki Municipal Hospital, TOYOTA Memorial Hospital, Okazaki Municipal Hospital, Konan Kosei Hospital, National Mie Central Medical Center, Ise Red Cross Hospital, Yokkaichi Municipal Hospital, Otsu Red Cross Hospital, Shiga University of Medical Science Hospital, Nagahama Red Cross Hospital, Uji Tokushukai Hospital, The Japan Baptist Hospital, Kyoto University, Kyoto Red Cross Daiichi Hospital, National Maizuru Medical Center, Fukuchiyama City Hospital, Kyoto Prefectural University of Medicine Hospital, Kyoto City Hospital, Mitsubishi Kyoto Hospital, Yodogawa Christian Hospital, Osaka Medical Center and Research Institute for Maternal and Child Health, Osaka University, Takatsuki General Hospital, Kansai Medical University, Osaka City General Hospital, Osaka City Sumiyoshi Hospital, Aizenbashi Hospital, Toyonaka Municipal Hospital, National Cerebral and Cardiovascular Center, Kitano Hospital, Saiseikai Suita Hospital, Chifune Hospital, Bellland General Hospital, Rinku General Medical Center, Osaka Red Cross Hospital, Yao Municipal Hospital, Osaka General Medical Center, Osaka City University, Hyogo Prefectural Kobe Children's Hospital, Kobe University, Kakogawa West City Hospital, Saiseikai Hyogoken Hospital, Kobe City Medical Center General Hospital, Hyogo College of Medicine Hospital, Himeji Red Cross Hospital, Toyooka Public Hospital, Hyogo Prefectural Awaji Medical Center, Nara Medical University, Wakayama Medical University, Tottori Prefectural Central Hospital, Tottori University, Shimane Prefectural Central Hospital, Matsue Red Cross Hospital, Kurashiki Central Hospital, Tsuyama Central Hospital, Kawasaki Medical School Hospital, National Hospital Organization Okayama Medical Center, Okayama Red Cross Hospital, Hiroshima City Hiroshima Citizens Hospital, Hiroshima Prefectural Hospital, Hiroshima University, Tsuchiya General Hospital, National Hospital Organization Kure Medical Center, Yamaguchi University, Yamaguchi Grand Medical Center, Tokushima University, Tokushima Municipal Hospital, Kagawa University, National Hospital Organization Kagawa Children's Hospital, Matsuyama Red Cross Hospital, Ehime Prefectural Central Hospital, Kochi Health Science Center, St.Mary's Hospital, National Kyushu Medical Center, Kurume University, Kitakyushu Municipal Medical Center, University of Occupational and Environmental Health, Fukuoka University, Kyushu University, lizuka Hospital, National Hospital Organization Kokura Medical Center, National Hospital Organization Saga Hospital, National Hospital Organization Nagasaki Medical Center, Kumamoto City Hospital, Kumamoto University, Oita Prefectural Hospital, Almeida Memorial Hospital, Nakatsu Municipal Hospital, Miyazaki University, National Hospital Organization Miyakonojo Medical Center, Kagoshima City Hospital, Imakiire General Hospital, Okinawa Prefectural Nanbu Medical Center \& Children's Medical Center, Okinawa Prefectural Chubu Hospital, Naha City Hospital, Okinawa Red Cross Hospital.

Contributors All authors contributed to the following; All authors meet all criteria of ICMJE.

Funding The authors have not declared a specific grant for this research from any funding agency in the public, commercial or not-for-profit sectors.

Competing interests None declared.

Patient consent for publication Not required.

Ethics approval The Internal Review Board of Tokyo Women's Medical University UMIN 000006961.

Provenance and peer review Not commissioned; externally peer reviewed.

Data availability statement All data relevant to the study are included in the article or uploaded as supplementary information.

Open access This is an open access article distributed in accordance with the Creative Commons Attribution Non Commercial (CC BY-NC 4.0) license, which permits others to distribute, remix, adapt, build upon this work non-commercially, and license their derivative works on different terms, provided the original work is properly cited, appropriate credit is given, any changes made indicated, and the use is non-commercial. See: http://creativecommons.org/licenses/by-nc/4.0/.

\section{REFERENCES}

1. Kim SY, Kotelchuck M, Wilson HG, et al. Prevalence of adverse pregnancy outcomes, by maternal diabetes status at first and second deliveries, Massachusetts, 1998-2007. Prev Chronic Dis $2015 ; 12$.
2. Michael Weindling A. Offspring of diabetic pregnancy: short-term outcomes. Semin Fetal Neonatal Med 2009;14:111-8.

3. Kodama Y, Sameshima H, Ohashi M, et al. Impact of new gestational diabetes mellitus criteria on stillbirth: a regional population-based study in Japan. J Obstet Gynaecol Res 2013;39:1242-5.

4. Li S, Zhang M, Tian $\mathrm{H}$, et al. Preterm birth and risk of type 1 and type 2 diabetes: systematic review and meta-analysis. Obes Rev 2014;15:804-11.

5. Zhu Y, Zhang C. Prevalence of gestational diabetes and risk of progression to type 2 diabetes: a global perspective. Curr Diab Rep 2016;16:7.

6. Matsushita E, Matsuda Y, Makino Y, et al. Risk factors associated with preterm delivery in women with pregestational diabetes. $J$ Obstet Gynaecol Res 2008;34:851-7.

7. Isayama T, Lee SK, Mori R, et al. Comparison of mortality and morbidity of very low birth weight infants between Canada and Japan. Pediatrics 2012;130:e957-65.

8. Boghossian NS, Hansen NI, Bell EF, et al. Outcomes of extremely preterm infants born to insulin-dependent diabetic mothers. Pediatrics 2016;137. doi:10.1542/peds.2015-3424

9. Rehan VK, Moddemann D, Casiro OG. Outcome of very-low-birthweight $(<1,500$ grams) infants born to mothers with diabetes. Clin Pediatr 2002;41:481-91.

10. Bental $Y$, Reichman $B$, Shiff $Y$, et al. Impact of maternal diabetes mellitus on mortality and morbidity of preterm infants (24-33 weeks' gestation). Pediatrics 2011;128:e848-55.

11. Grandi C, Tapia JL, Cardoso VC. Impact of maternal diabetes mellitus on mortality and morbidity of very low birth weight infants: a multicenter Latin America study. J Pediatr 2015;91:234-41.

12. Persson M, Shah PS, Rusconi F, et al. Association of maternal diabetes with neonatal outcomes of very preterm and very lowbirth-weight infants: an international cohort study. JAMA Pediatr 2018;172:867.

13. Metzger BE, Gabbe SG, Persson B, et al. International association of diabetes and pregnancy study groups recommendations on the diagnosis and classification of hyperglycemia in pregnancy. Diabetes Care 2010;33:e98-82.

14. Morikawa M, Yamada T, Yamada T, et al. Change in the number of patients after the adoption of IADPSG criteria for hyperglycemia during pregnancy in Japanese women. Diabetes Res Clin Pract 2010;90:339-42.

15. Sugiyama T, Kusaka H, Sagawa $\mathrm{S}$, et al. Report of collaborative study on the screening tests of gestational diabetes mellitus. Tounyou-byo to Ninshin 2006;6:7-12.

16. Shah PS, Lui K, Sjörs G, et al. Neonatal outcomes of very low birth weight and very preterm neonates: an international comparison. $J$ Pediatr 2016;177:144-52.

17. Kusuda S, Fujimura M, Sakuma I, et al. Morbidity and mortality of infants with very low birth weight in Japan: center variation. Pediatrics 2006;118:e1130-8.

18. Tokumasu H, Tokumasu S, Kawakami K. Impact of pre-eclampsia in extremely premature infants: population-based study. Pediatr Int 2016:58:578-83.

19. Papile LA, Burstein J, Burstein R, et al. Incidence and evolution of subependymal and intraventricular hemorrhage: a study of infants with birth weights less than 1,500 GM. J Pediatr 1978;92:529-34.

20. Itabashi K, Fujimura M, Kusuda S, et al. A modified version of new Japanese neonatal anthropometric charts for gestational age at birth. J Jpn Pediatr Soc 2010;114:1771-806.

21. Itabashi K, Miura F, Uehara R, et al. New Japanese neonatal anthropometric charts for gestational age at birth. Pediatr Int 2014;56:702-8

22. Azad MB, Moyce BL, Guillemette L, et al. Diabetes in pregnancy and lung health in offspring: developmental origins of respiratory disease. Paediatr Respir Rev 2017;21:19-26.

23. Piper JM, Xenakis EM, Langer O. Delayed appearance of pulmonary maturation markers is associated with poor glucose control in diabetic pregnancies. J Matern Fetal Med 1998;7:148-53.

24. Miakotina OL, Goss KL, Snyder JM. Insulin utilizes the PI 3-kinase pathway to inhibit SP-A gene expression in lung epithelial cells. Respir Res 2002;3:27.

25. Hay WW. Care of the infant of the diabetic mother. Curr Diab Rep 2012;12:4-15.

26. Anadkat JS, Kuzniewicz MW, Chaudhari BP, et al. Increased risk for respiratory distress among white, male, late preterm and term infants. J Perinatol 2012;32:780-5.

27. Fong A, Serra A, Herrero T, et al. Pre-gestational versus gestational diabetes: a population based study on clinical and demographic differences. J Diabetes Complications 2014;28:29-34. 